\title{
Extractive bioconversion of cyclodextrins by Bacillus cereus cyclodextrin glycosyltransferase in aqueous two-phase system
}

\begin{abstract}
An extractive bioconversion with Bacillus cereus cyclodextrin glycosyltransferase (CGTase, EC 2.4.1.19) in aqueous two-phase system (ATPS) was investigated for the synthesis and recovery of cyclodextrins (CDs). Optimum condition for the extractive bioconversion of CDs was achieved in ATPS consisted of 7.7\% (w/w) polyethylene glycol (PEG) 20,000 and 10.3\% (w/w) dextran T500 with volume ratio (VR) of 4.0. Enzymatic conversion of starch occurred mainly in dextran-rich bottom phase whereas the product, CDs was transferred to top phase and a higher partition coefficient of CDs was achieved. Repetitive batch of CDs synthesis was employed by replenishment of the top phase components and addition of starch every 8 h. An average total CDs concentration of $13.7 \mathrm{mg} / \mathrm{mL},(4.77 \mathrm{mg} / \mathrm{mLU}-\mathrm{CD}, 5.02 \mathrm{mg} / \mathrm{mL}$-CD and $3.91 \mathrm{mg} / \mathrm{mL}-\mathrm{CD}$ ) was recovered in the top phase of PEG 20,000/dextran T500 ATPS. This study showed the effectiveness of ATPS application in extractive bioconversion of CDs synthesis with B. cereus CGTase.
\end{abstract}

Keyword: Aqueous two-phase system; Extractive bioconversion; Cyclodextrin; Bacillus cereus; Cyclodextrin glycosyltransferase 